\title{
Today's common sense in science can be changed tomorrow: STEM education
}

\author{
yoshiyasu takefuji ${ }^{1}$ \\ ${ }^{1}$ Affiliation not available
}

September 21, 2020

\section{Keywords:}

STEM education; scientific laws; scientific public policy

\section{ABSTRACT:}

STEM (Science, Technology, Engineering, Mathematics) education plays a key role in the sustained growth and stability of the economy, and is a critical component to helping any country win the future ${ }^{1}$. STEM education also creates critical thinkers, increases science literacy, and enables the next generation of innovators ${ }^{1}$. Although mathematics is robust, scientific laws are statements based on repeated experiments or observations that describe or predict a range of natural phenomena. Therefore, different observations may produce a new scientific law which may contradict with the conventional scientific laws. This short paper introduces two examples for validating the claim. Teachers must inform all students that scientific laws are not always correct but correct only under the fixed observations. In other words, in the future the current common sense in science can be changed tomorrow.

\section{TEXT}

One example is how to protect ourselves against covid-19. Although droplet protection was initially informed by WHO against covid-19, the airborne protection should have been proposed based on the past lessons from SARS and MERS. Another example is Pouillet's law or Ohm's law. Based on Pouillet's law, the electrical resistance is proportional to electrical resistivity, the length of the specimen. According to the conductance quantum, the resistance of an atomic conductor does not scale proportional to length which contradicts with Pouillet's law.

Although WHO (world health organization) should be a trusted organization, WHO has informed us optimistic protections against covid-19. WHO gave advices on wearing masks to protect against covid-19 in January $2020^{2}$. WHO stated that the virus can be spread via infectious droplets or through touching contaminated surfaces in April $2020^{3}$. WHO gave the contradictory message in June 2020 "Non-medical, fabric masks are being used by many people in public areas, but there has been limited evidence on their effectiveness and WHO does not recommend their widespread use among the public for control of COVID-19" ${ }^{4}$. In July 2020, WHO finally announced that airborne precautions are needed against covid- $19^{5}$. WHO should have known the following fact long time ago that "droplet precautions should be added to standard precautions when providing care to all patients with symptoms of acute respiratory infection. Contact precautions and eye protection should be added when caring for suspected or confirmed cases of MERS. Airborne precautions should be applied when performing aerosol-generating procedures" ${ }^{\prime 7,7}$. Based on the past lessons of SARS and MERS, pessimistic protections instead of optimistic protections should be announced by the trusted scientific communities including WHO against the infection diseases.

Based on Pouillet's law, the electrical resistance of a uniform specimen of the material is proportional to 
electrical resistivity, the length of the specimen, and the inverse of the cross-sectional area of the specimen respectively. $\mathrm{Au}$ (gold) can be stretched into conducting chains of individual atoms ${ }^{9}$. After a new discovery of the conductance quantum, we understand that the resistance of an atomic conductor does not scale proportional to length ${ }^{9}$. This means that scientific information is not robust but tentative. Science should be based on replacing expired facts and evidences with new ones.

\section{CONCLUSION}

Teachers of STEM education must know two facts. One is that the current common sense in science can be changed tomorrow. Another is that the lay public including parents does not know that known science laws may be incorrect under new observations. Scientific laws are not always true but true under the fixed conventional observations. Once scientific laws (claims) were changed, the lay public may lose trust in science. Once the public trust is lost, rebuilding trust in science is really hard. Therefore, public science policy or pubic STEM policy should be addressed: all scientific laws (claims) are temporal and they may be changed tomorrow. Today's common sense can be changed tomorrow.

This research did not receive any specific funding. The authors declare no conflict of interest. The author has read the manuscript and has approved this submission. Ethical statement is not available. Consent statement is not available.

References:

1. https://www. engineeringforkids . com/about/news/2016/february/why-is-stem-education-soimportant-/

2. https://www. who.int/docs/default-source/documents/advice-on-the-use-of-masks-2019ncov.pdf

3. Advice on the use of masks in the context of COVID-19, April 6, WHO

https://apps.who.int/iris/handle/10665/331693

4. Coronavirus disease (COVID-19) advice for the public: When and how to use masks 23 June 2020

https://www . afro. who. int/news/coronavirus-disease-covid-19-advice-public-when-and-howuse-masks

5. Transmission of SARS-CoV-2: implications for infection prevention precautions 9 July 2020 WHO

https://www . who.int/news-room/commentaries/detail/transmission-of-sars-cov-2-

implications-for-infection-prevention-precautions

6. https://www.who.int/csr/disease/coronavirus_infections/faq/en/

7. https://www.who.int/csr/disease/coronavirus_infections/MERSCov_WHO_KSA_Mission_Jun13_ $\cdot \mathrm{pdf}$

8. https://www.who.int/csr/sars/WHOconsensus.pdf?ua=1

9. J Chen, MA Reed, AM Rawlett, JM Tour: "Large on-off ratios and negative differential resistance in a molecular electronic device," Science 286 (1999) 1550- 1552. 